\title{
ESTERIFICAÇÃO DE ÁCIDOS GRAXOS UTILIZANDO ZIRCÔNIA SULFATADA E COMPÓSITOS CARVÃO ATIVADO/ZIRCÔNIA SULFATADA COMO CATALISADORES
}

\author{
Sarah S. Brum*, Valéria C. dos Santos, Priscila Destro e Mário César Guerreiro \\ Departamento de Química, Universidade Federal de Lavras, CP 3037, 37200-000 Lavras - MG, Brasil
}

Recebido em 25/10/10; aceito em 3/5/11; publicado na web em 29/6/11

\begin{abstract}
ESTERIFICATION OF FATTY ACIDS USING SULFATED ZIRCONIA AND COMPOSITES ACTIVATED CARBON / SULFATED ZIRCONIA CATALYSTS. In this work sulfated zirconia (SZr) and activated carbon/SZr composites produced by impregnation method with or without heating treatment step (CABC/SZr-I and CABC/SZr-I SC) and by the method of synthesis of SZr on the carbon (CABC/SZr-S) was used as catalysts in the esterification reactions of fatty acids. The SZr presented very active, conversions higher than $90 \%$ were obtained after $2 \mathrm{~h}$ of reaction. The activity of the composite CABC/SZr-I20\%SC was up to $92 \%$, however, this was directly related to time and temperature reactions. CABC/SZr-I and CABC/SZr-S were less active in esterification reactions, what could be attributed to its low acidity.
\end{abstract}

Keywords: acid catalysis; esterification; biodiesel.

\section{INTRODUÇÃO}

A busca por melhorias no processo de produção de biodiesel tem apontado para a síntese de novos catalisadores mais ativos e robustos como uma das principais alternativas. Atualmente, a catálise básica homogênea tem sido o processo industrial mais utilizado em todo mundo para a produção de biodiesel, entretanto, a utilização de catalisadores básicos exige que o óleo empregado seja refinado (menos que $1 \%$ de acidez) e todos os reagentes empregados sejam anidros. Do contrário, parte do catalisador será utilizada para neutralizar os ácidos graxos presentes no óleo, gerando grandes quantidades de sabões. ${ }^{1}$ É devido a esses fatores que, mesmo com o processo industrial estabelecido, ainda existe uma grande procura por um processo ideal, o qual consiga transformar qualquer matéria-prima, independente de sua origem (animal, vegetal, residual ou não). ${ }^{2}$

Uma alternativa ao processo de produção de biodiesel é a catálise ácida. A grande vantagem da catálise ácida é que ésteres monoalquílicos (biodiesel) podem ser obtidos tanto por reações de esterificação dos ácidos graxos como por reações de transesterificação dos triglicerídeos, possibilitando a utilização de matérias-primas de baixa qualidade, as quais contêm grandes quantidades de ácidos graxos livres e, portanto, mais viáveis economicamente. Nesse contexto, os catalisadores heterogêneos, principalmente sólidos ácidos, destacamse por serem menos corrosivos e, especialmente, por poderem ser reutilizados e usados em processos contínuos. ${ }^{3}$

Diversos catalisadores sólidos ácidos têm sido estudados em reações de esterificação de ácidos graxos e transesterificação de triglicerídeos, tais como os de titânia sulfatada, ${ }^{4}$ poliestireno sulfonado, ${ }^{5}$ resinas Amberlyst ${ }^{\circledR} 15,{ }^{6}$ Nafion, ${ }^{7}$ heteropoliácidos heterogêneos, ${ }^{8}$ entre outros. Entretanto, desde que Hino e Arata ${ }^{9}$ mostraram que o butano poderia ser transformado em isobutano em temperatura ambiente utilizando zircônia sulfatada, tem sido dada grande atenção para esse catalisador. ${ }^{10,11}$ Recentemente, alguns estudos têm mostrado que, por apresentar características superácidas, ela é capaz de catalisar inúmeras reações de interesse industrial, tais como isomerização de

*e-mail: sarahsbrum@yahoo.com.br hidrocarbonetos, craqueamento, alquilação e eterificação. ${ }^{12-14}$

Diversos métodos de preparação de zircônia sulfatada são relatados na literatura, utilizando-se diferentes precursores e agentes de sulfatação. Mas, há um consenso de que, para se obter maiores quantidades de sítios ácidos, são necessárias temperaturas de calcinação em torno de 600 a $650{ }^{\circ} \mathrm{C}$. No entanto, nessas temperaturas, a zircônia sulfatada apresenta baixa área superficial.

Alguns trabalhos têm mostrado que é possível melhorar as propriedades texturais da zircônia sulfatada dispersando-a em suportes como nanotubos de carbono e sílicas. ${ }^{15,16}$ Neste contexto, a utilização de carvão ativado como suporte surge como uma alternativa promissora, na medida em que seria possível agregar características da zircônia sulfatada, como a alta acidez, às do carvão ativado, tais como elevada área superficial, estabilidade térmica e tolerância à água.

Dessa forma, os objetivos do trabalho foram sintetizar a zircônia sulfatada, utilizando uma rota sintética alternativa, livre de solventes e precipitação; produzir compósitos catalisadores de zircônia sulfatada, usando o carvão ativado como suporte e, testar os catalisadores produzidos em reações de esterificação de ácidos graxos.

\section{PARTE EXPERIMENTAL}

\section{Produção do carvão ativado a partir da borra de café}

Para produção dos carvões ativados foi utilizada como precursora a borra de café, cedida pela Indústria Café Solúvel Brasília S.A. (Varginha, MG).

A borra de café foi impregnada com uma solução de $\mathrm{K}_{2} \mathrm{CO}_{3}$, na proporção de $1: 1(\mathrm{~m} / \mathrm{m})$ e colocada em estufa, a $110{ }^{\circ} \mathrm{C}$, por $24 \mathrm{~h} . \mathrm{O}$ material impregnado foi submetido a tratamento térmico em forno tubular sob atmosfera inerte de $\mathrm{N}_{2}$, com fluxo de $180 \mathrm{~mL} \mathrm{~min}^{-1}$ e aquecimento com rampa de temperatura de $10{ }^{\circ} \mathrm{C} \mathrm{min}^{-1}$, com temperatura inicial de $50{ }^{\circ} \mathrm{C}$ até a temperatura final de $800^{\circ} \mathrm{C}$, permanecendo por $2 \mathrm{~h}$ nesta temperatura. Após a carbonização do material, o mesmo foi lavado com água quente (aproximadamente $80^{\circ} \mathrm{C}$ ) para desobstrução dos poros formados. Em seguida, o carvão foi lavado com água destilada até $\mathrm{pH}$ neutro e seco em estufa, a $60^{\circ} \mathrm{C}$. 


\section{Síntese da zircônia sulfatada (SZr)}

A zircônia sulfatada (SZr) foi sintetizada por um método seco descrito por Sun et al. ${ }^{17}$ a partir de uma mistura de $10 \mathrm{~g}(31 \mathrm{mmol}) \mathrm{de}$ $\mathrm{ZrOCl}_{2} .8 \mathrm{H}_{2} \mathrm{O}$ e $24,6 \mathrm{~g}$ (186 mmol) de $\left(\mathrm{NH}_{4}\right)_{2} \mathrm{SO}_{4}$ (razão molar 1:6). Os sais foram homogeneizados, durante $20 \mathrm{~min}$, em um almofariz de ágata. A mistura foi deixada em repouso à temperatura ambiente por $18 \mathrm{~h}$ e, em seguida, calcinada a $600{ }^{\circ} \mathrm{C}$, por $5 \mathrm{~h}$.

\section{Produção dos compósitos carvão ativado/zircônia sulfatada}

Para a produção dos compósitos foram utilizados dois processos:

\section{Método de impregnação}

Os carvões ativados produzidos a partir da borra de café foram impregnados com uma solução contendo $20 \%$ de SZr (em relação à massa de carvão ativado), por $24 \mathrm{~h}$, a $100{ }^{\circ} \mathrm{C}$. Após esse período, o carvão impregnado foi calcinado a $600{ }^{\circ} \mathrm{C}$, por $5 \mathrm{~h}$, sob fluxo de nitrogênio. Este material foi designado como CABC/SZr-I20\%. Para se verificar a influência da calcinação, um material foi produzido sem a etapa de calcinação a $600{ }^{\circ} \mathrm{C}$ e designado CABC/SZr-I20\% SC.

\section{Método de síntese da SZr sobre o CABC}

Para a produção desse compósito, a zircônia sulfatada foi sintetizada juntamente com o carvão ativado da borra de café. Para isso, o CABC foi homogeneizado com o oxicloreto de zircônio $\left(\mathrm{ZrOCl}_{2} \cdot 8 \mathrm{H}_{2} \mathrm{O}\right)$ e com o sulfato de amônio $\left(\left(\mathrm{NH}_{4}\right)_{2} \mathrm{SO}_{4}\right)$, seguindo o mesmo procedimento utilizado na síntese da $\mathrm{SZr}$. Utilizaram-se as proporções $3 \mathrm{~g}$ de CABC para $0,4936 \mathrm{~g}$ de $\mathrm{ZrOCl}_{2} \cdot 8 \mathrm{H}_{2} \mathrm{O}$ e $1,216 \mathrm{de}$ $\left(\mathrm{NH}_{4}\right)_{2} \mathrm{SO}_{4}(\mathrm{CABC} / \mathrm{SZr}-\mathrm{S} 20 \%)$.

\section{Caracterização dos materiais}

Os materiais produzidos foram caracterizados por adsorção/dessorção de $\mathrm{N}_{2}$ a $77 \mathrm{~K}$, usando-se o instrumento Autosorb-1 da Quantachrome. A adsorção de $\mathrm{N}_{2}$ foi analisada por meio da equação BET. A fase cristalina foi obtida por difratometria de Raios-X, utilizando-se um difratômetro da Rigaku com variação angular de $20^{\circ}-80^{\circ}(2 \theta)$, radiação de $\operatorname{CoK} \alpha(\lambda=1,78897 \AA)$ e velocidade de exposição de $1^{\circ}$ $\mathrm{min}^{-1}$. As análises termogravimétricas foram realizadas utilizando-se um aparelho analisador termomecânico Shimadzu DTG 60-AH. As amostras foram aquecidas de $25 \mathrm{a} 1.100^{\circ} \mathrm{C}$, com taxa de aquecimento $10{ }^{\circ} \mathrm{C} \min ^{-1}$ e fluxo de ar de $30 \mathrm{~mL} \mathrm{~min}^{-1}$.

\section{Teste de acidez}

A acidez dos catalisadores foi obtida por meio de titulação. Para isso, $100 \mathrm{mg}$ do material foram deixados em contato com $0,020 \mathrm{~L}$ de $\mathrm{NaOH} 0,1 \mathrm{~mol} \mathrm{~L}^{-1}$, por $3 \mathrm{~h}$, sob leve agitação. Após esse período, foram retiradas alíquotas da solução básica que foram tituladas com $\mathrm{HCl} 0,1 \mathrm{~mol} \mathrm{~L}^{-1}$, para se verificar a quantidade de $\mathrm{NaOH}$ que reagiu com o material. A acidez foi determinada em mmol de $\mathrm{H}^{+}$por grama de material.

\section{Testes catalíticos}

\section{Reação de esterificação de ácido láurico}

Foram realizadas em sistema fechado. Utilizaram-se tubos de vidro com tampa de rosca, os quais foram colocados em um reator com agitação elíptica e controle de temperatura. As reações foram conduzidas a 70 e $100{ }^{\circ} \mathrm{C}$, sob agitação de $300 \mathrm{rpm}$, durante $24 \mathrm{~h}$. Para os ensaios, a proporção ácido graxo:metanol foi de 1:50 e diferentes concentrações de catalisador foram testadas.
Um estudo cinético também foi realizado; foram retiradas alíquotas em 0,$5 ; 1 ; 2 ; 3 ; 4$ e $6 \mathrm{~h}$, diluídas em hexano e analisadas por cromatografia em fase gasosa.

\section{Reação de esterificação de óleo de soja/20\% ácido láurico}

As reações de esterificação do óleo de soja/20\% ácido láurico foram desenvolvidas da mesma forma relatada para a esterificação do ácido láurico. Os ensaios foram realizados à temperatura de 100 ${ }^{\circ} \mathrm{C}$, nas seguintes proporções: $0,334 \mathrm{~mL}$ de metanol $(8,25 \mathrm{mmol})$, 0,1837 g de ácido láurico (0,9 mmol) e 0,92 g de óleo de soja comercial. Foi realizado um estudo cinético, no qual alíquotas foram retiradas em 0,$5 ; 1 ; 2 ; 3 ; 4$ e 6 h, diluídas em hexano e analisadas por cromatografia gasosa.

\section{Quantificação dos ésteres metílicos por cromatografia gasosa}

A porcentagem de conversão de ácido láurico em laureato de metila foi determinada por cromatografia em fase gasosa. As análises foram realizadas utilizando-se aparelho GC-2010 Shimadzu, equipado com um detector de ionização de chama (FID), um injetor automático e uma coluna SP $2560(100 \mathrm{~m} \mathrm{X} \mathrm{0,25mm} \mathrm{X} \mathrm{0,20} \mathrm{\mu m).}$ A temperatura inicial do forno foi de $150{ }^{\circ} \mathrm{C}$, que permaneceu por 5 min. Em seguida, a temperatura foi elevada para $240{ }^{\circ} \mathrm{C}$, com uma taxa de aquecimento de $15{ }^{\circ} \mathrm{C}$ por min, permanecendo nessa temperatura por mais $5 \mathrm{~min}$.

\section{RESULTADOS E DISCUSSÃO}

\section{Adsorção/dessorção de $\mathbf{N}_{2}$}

Na Figura 1a são mostradas as isotermas de adsorção/dessorção de nitrogênio à $77 \mathrm{~K}$ para $\mathrm{SZr}$ e $\mathrm{ZrO}_{2}$. A forma da isoterma de adsorção pode fornecer informações qualitativas preliminares sobre o mecanismo de adsorção e da estrutura porosa dos materiais.

De acordo com a classificação de isotermas propostas por BET (Figura 1a), o $\mathrm{ZrO}_{2}$ apresenta uma isoterma do tipo II, que é característica de processos de adsorção em sólidos não porosos ou macroporos ( $>>500 \AA$ ) , ocorrendo adsorção em multicamadas. Na isoterma da SZr, observa-se a ocorrência do fenômeno de histerese, que é a não sobreposição da curva de adsorção e dessorção, devido à diferença no processo de evaporação e condensação, típico de sólidos do tipo IV. Nesse tipo de isoterma, as maiores adsorções ocorreram em maiores pressões relativas, o que caracteriza a formação de sólidos mesoporosos.

Observa-se que a incorporação de sulfatos no óxido de zircônio resultou em um aumento de área de 16 para $65 \mathrm{~m}^{2} \mathrm{~g}^{-1}$. O valor de área superficial encontrado neste trabalho está dentro da faixa dos valores encontrados na literatura. Sun et al. ${ }^{17}$ obtiveram um valor mais alto, de $165,2 \mathrm{~m}^{2} \mathrm{~g}^{-1}$. No entanto, Garcia, ${ }^{18}$ utilizando a mesma rota sintética, obteve valor de área bem menor para a zircônia sulfatada, 12,6 $\mathrm{m}^{2} \mathrm{~g}^{-1}$. Já Du et al., ${ }^{19}$ sintetizando a zircônia sulfatada pelo método convencional, obtiveram um valor próximo ao encontrado neste trabalho, $75 \mathrm{~m}^{2} \mathrm{~g}^{-1}$.

Na Figura 1b estão presentes as isotermas de adsorção/dessorção de $\mathrm{N}_{2}$ para o CABC e para os compósitos. Observa-se que todos os materiais apresentam isoterma do tipo I. Essa forma é típica de sólidos microporosos, ou seja, com tamanho de poro abaixo de $20 \AA$. A área superficial do CABC foi de $900 \mathrm{~m}^{2} \mathrm{~g}^{-1}$. A incorporação de $5 \%$ de sulfato resultou em uma pequena diminuição de área, 840 $\mathrm{m}^{2} \mathrm{~g}^{-1}$ para o CABC/SZr-I5\% e $780 \mathrm{~m}^{2} \mathrm{~g}^{-1}$ para o CABC/SZr-S5\%. $\mathrm{O}$ aumento da quantidade de $\mathrm{SZr}$ na superfície do carvão ativado não implicou em uma diminuição de área. Áreas semelhantes foram encontradas para os materiais com $20 \%$ de SZr. Os resultados são mostrados na Tabela 1. 

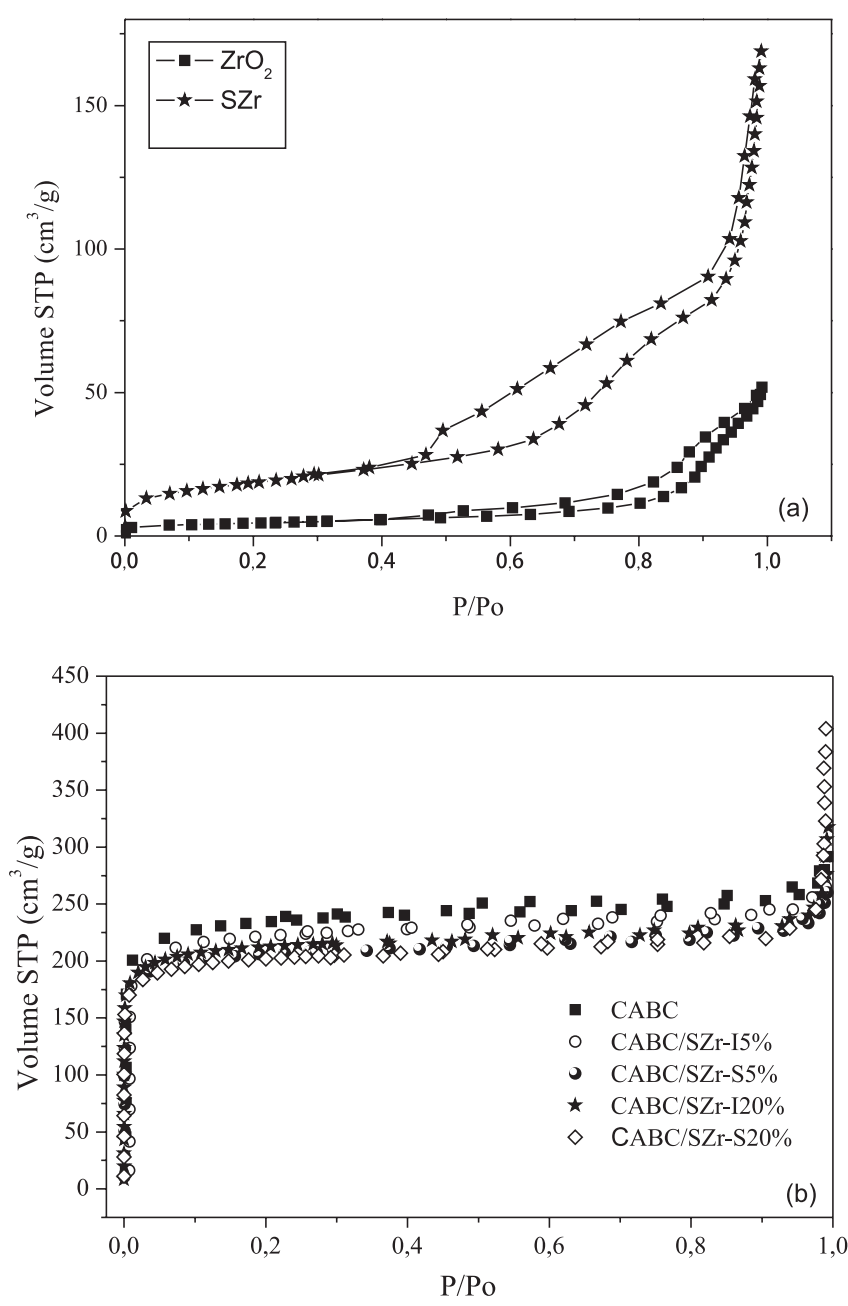

Figura 1. Isotermas de adsorção/dessorção de $N_{2}$ para (a) zircônia sulfatada $(\mathrm{SZr})$ e zircônia $\left(\mathrm{ZrO}_{2}\right)$ e (b) carvão ativado da borra de café $(\mathrm{CABC})$ e compósitos CABC/SZr-I e S)

Tabela 1. Valores de área superficial (BET) e volumes de poro para os catalisadores

\begin{tabular}{lcccc}
\hline Catalisador & $\begin{array}{c}\text { Área } \\
\left(\mathrm{m}^{2} \mathrm{~g}^{-1}\right)\end{array}$ & $\begin{array}{c}\mathrm{V}_{\text {Total }} \\
\left(\mathrm{cm}^{3} \mathrm{~g}^{-1}\right)\end{array}$ & $\begin{array}{c}\mathrm{V}_{\text {meso }} \\
\left(\mathrm{cm}^{3} \mathrm{~g}^{-1}\right)\end{array}$ & $\begin{array}{c}\mathrm{V}_{\text {micro }} \\
\left(\mathrm{cm}^{3} \mathrm{~g}^{-1}\right)\end{array}$ \\
\hline $\mathrm{ZrO}_{2}$ & 16 & - & - & - \\
$\mathrm{SZr}$ & 65 & - & - & - \\
$\mathrm{CABC}$ & 900 & 0,41 & 0,04 & 0,37 \\
$\mathrm{CABC/SZr-I5 \%}$ & 840 & 0,39 & 0,03 & 0,36 \\
$\mathrm{CABC/SZr-S5 \%}$ & 780 & 0,37 & 0,05 & 0,32 \\
CABC/SZr-I20\% & 822 & 0,38 & 0,06 & 0,32 \\
CABC/SZr-S20\% & 775 & 0,37 & 0,07 & 0,31 \\
\hline
\end{tabular}

\section{Análise por difratometria de raios-X}

A zircônia sulfatada pode ser cristalina ou amorfa. Quando cristalina, pode existir em três formas: tetragonal, monoclínica e cúbica.

Na Figura 2 estão presentes os difratogramas de raios X da SZr e dos compósitos CABC/SZr.

Analisando-se o difratograma da SZr, observa-se a formação de um material com fase predominantemente tetragonal. ${ }^{20} \mathrm{~A}$ fase tetragonal é bastante desejável, já que um consenso é o de que a presença

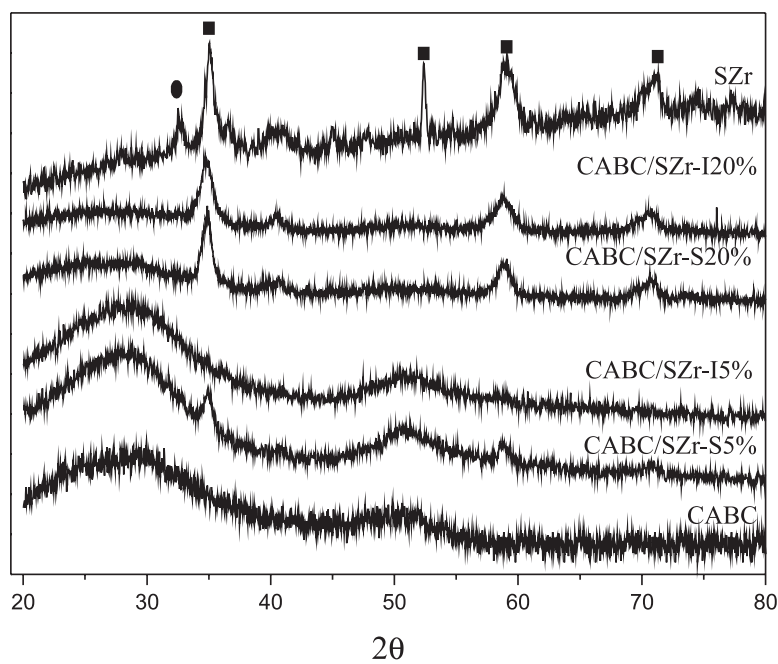

Figura 2. Difratogramas de raios $X(\bigcirc$ fase monoclínica e $\mathbf{\square}$ fase tetragonal)

de uma maior quantidade de fase tetragonal implica em uma maior acidez para a zircônia sulfatada e, consequentemente, uma maior atividade catalítica. ${ }^{13,17,18,21}$

Segundo Noda et al., ${ }^{21}$ a zircônia sulfatada amorfa apresenta a maioria dos grupos sulfatos no interior da estrutura, com um caráter iônico, formando sítios menos ácidos. Após a cristalização e a formação de fase tetragonal, o sulfato é deslocado para a superfície, dando origem a duas ligações $\mathrm{S}=\mathrm{O}$ (com um caráter mais covalente), no caso da amostra desidratada, sendo responsável pela formação de sítios de Brönsted e de Lewis altamente ácidos. A fase tetragonal é uma fase metaestável e, dependendo da temperatura de calcinação ( $>650$ ${ }^{\circ} \mathrm{C}$ ) do material, podem ocorrer perdas do sulfato que acarretam na formação da fase monoclínica, mais estável, mas, consequentemente, menos ácida.

Ainda na Figura 2, comparando os difratogramas da SZr, CABC com os compósitos nota-se o aumento da cristalinidade com o aumento de SZr no material.

O compósito produzido pelo método de impregnação com $5 \%$ de SZr (CABC/SZr-I5\%) não apresentou difrações referentes a SZr, entretanto, para o compósito produzido com a mesma quantidade de $\mathrm{SZr}$, preparada pelo método a seco de síntese, duas difrações foram observadas em $2 \theta=34,6$ e 59,3 .

Os difratogramas dos materiais preparados contendo $20 \%$ de SZr preparados pelo método de síntese a seco (CABC/SZr-S20\%) e pelo método de impregnação (CABC/SZr-I20\%) não apresentaram diferenças perceptíveis, contendo difrações em 2 = 34,6; 59,3 e 71,6, todas referentes à fase tetragonal.

A princípio, o método de síntese a seco apresenta a vantagem de ser mais rápido e simples quando comparado com o método de impregnação, no qual o material é produzido em duas etapas, a primeira com a produção da SZr e a segunda, a impregnação propriamente dita. No método de síntese a seco, a SZr é sintetizada diretamente no $\mathrm{CABC}$, minimizando o tempo e o custo do preparo.

\section{Análise termogravimétricas}

As curvas de TG-DTG das amostras SZr, CABC e compósitos $\mathrm{CABC} / \mathrm{SZr}$ podem ser observadas nas Figuras 3.

Para a $\mathrm{SZr}$, quatro zonas de perda de massas podem ser observadas. Nas temperaturas entre $25-210{ }^{\circ} \mathrm{C}$, a perda de $10 \%$ de massa é relativa a processos de desidratação e desidroxilação, respectivamente. As perdas de massas referentes ao grupo sulfato (34\%) são observadas entre as temperaturas $531-1.100^{\circ} \mathrm{C}$. Três picos podem ser considera- 


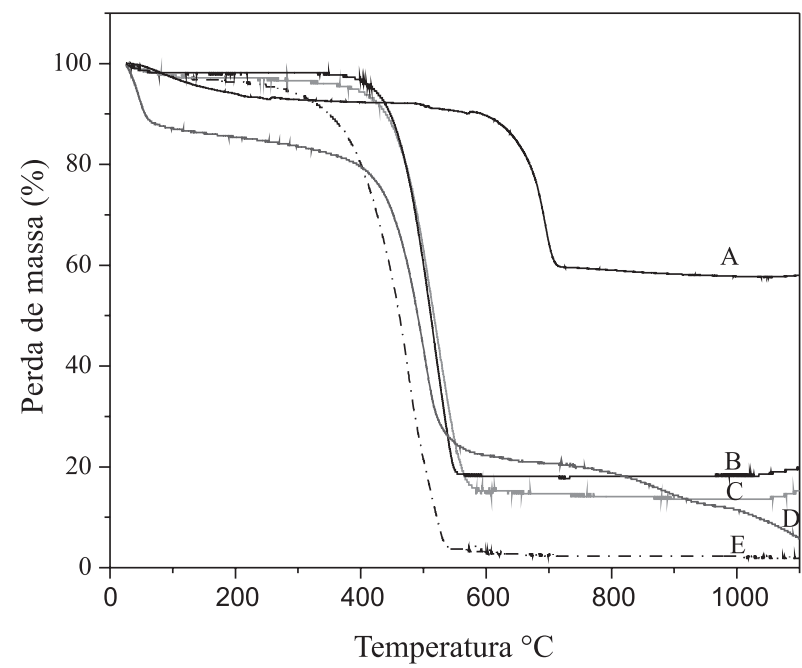

Figura 3. Curvas termogravimétricas das amostras: (A) zircônia sulfatada (SZr), (B) CABC sintetizado com $20 \%$ de $\mathrm{SZr}$ (CABC/SZrS20\%), (C) CABC impregnado com $20 \%$ de SZr (CABC/SZr-I20\%), (D) CABC impregnado com $20 \%$ de SZr sem calcinar (CABC/SZr$I 20 \% S C)$ e (E) carvão ativado da borra de café (CABC)

dos nesta região, devido à decomposição de espécies $\mathrm{SO}_{4}{ }^{-2} \mathrm{a} \mathrm{SO}_{3}$. O primeiro pouco intenso, referente à perda de $2 \%$, centrado na curva de DTG em $531{ }^{\circ} \mathrm{C}$, provavelmente devido ao início da decomposição dos grupos sulfatos menos estáveis; o segundo, mais intenso, centrado em $697{ }^{\circ} \mathrm{C}$, responsável por $30,5 \%$ de redução de massa e o último, mais amplo e pouco acentuado $(2,1 \%)$, centrado em torno de $905^{\circ} \mathrm{C}$. Garcia, ${ }^{18}$ utilizando a mesma rota sintética para a preparação da zircônia sulfatada, também encontrou altas perdas de massas referentes à decomposição dos grupos sulfatos, 40,7\%, em torno de 600 a 750 ${ }^{\circ} \mathrm{C}$. Noda et al., ${ }^{21}$ estudando a preparação de zircônias sulfatadas por diferentes rotas sintéticas, obtiveram perdas em torno de $20 \%$ nas mesmas faixas de temperatura. Entretanto, menores valores foram encontrados por Sun et al., ${ }^{17}$ cerca de 7,7\% de perda, referentes ao grupos sulfatos. Contudo, segundo os próprios autores, maiores perdas de massa relativas a grupos sulfatos podem ser relacionadas a maiores quantidades de sítios ácidos.

Correlacionando os resultados obtidos pelas análises termogravimétricas e por difratometria de raios $\mathrm{X}$, é interessante observar que a calcinação da amostra a $600{ }^{\circ} \mathrm{C}$ levou à formação de uma fase predominantemente tetragonal, temperatura limitante observada no termograma para a perda de sulfato.

Foi possível verificar que ocorreu a formação dos compósitos, comparando os seus termogramas com o do carvão CABC puro, os quais apresentam um perfil diferente. Para o CABC, verifica-se uma pequena perda de massa entre 25 a $100{ }^{\circ} \mathrm{C}(6 \%)$ referente à água de hidratação. A perda de massa de $92 \%$ ocorreu entre $260-550^{\circ} \mathrm{C}$, com um pico na curva de DTG centrado em $471^{\circ} \mathrm{C}$.

Os compósitos CABC-SZr-S20\% e CABC-SZr-I20\% obtiveram perdas de massas totais de aproximadamente 80 e $85 \%$, respectivamente, ambas menores que a do CABC $(98,2 \%)$, um indicativo da presença de SZr no material. Para esses materiais, o pico intenso centrado entre 503 e $506^{\circ} \mathrm{C}$ foi relacionado à perda de massa referente à decomposição do carvão. As perdas referentes aos grupos sulfatos foram atribuídas aos picos em torno de $596-577^{\circ} \mathrm{C}$, entretanto, para os dois materiais, uma perda de apenas 1,8 e 3,4\% foi observada, respectivamente.

Em contrapartida, para o compósito obtido sem a etapa de calcinação (CABC/SZr-I20\%SC), maiores perdas referentes ao sulfato foram alcançadas, em torno de 579 e $962{ }^{\circ} \mathrm{C}$, totalizando $10,8 \%$. Um ponto importante foi que, do total, $6,4 \%$ da massa foram perdidos em torno de $962{ }^{\circ} \mathrm{C}$. Ou seja, o compósito CABC/SZr-I20\%SC, além de apresentar maior número de grupos sulfatos que os outros compósitos, esses grupos são termicamente mais estáveis, principalmente quando comparados aos grupos sulfatos presentes na SZr.

Os compósitos, independente do processo de preparo, apresentaram perdas de massa referentes ao carvão e ao sulfato. No entanto, observa-se que a formação do compósito leva à perda de sulfatos em diferentes temperaturas, caracterizando a formação de um novo material com propriedades diferentes de seus precursores.

\section{Teste de acidez}

Os resultados de acidez dos materiais são apresentados na Tabela 2 .

Tabela 2. Valores de acidez dos materiais: zircônia sulfatada (SZr), zircônia $\left(\mathrm{ZrO}_{2}\right)$, carvão ativado da borra de café (CABC), CABC impregnado com $20 \%$ de SZr (CABC/SZr-I20\%), CABC sintetizado com 20\% de SZr (CABC/ SZr-S20\%), CABC impregnado com 20\% de SZr- sem calcinar (CABC/ SZr-I20\%SC) e nióbia

\begin{tabular}{|c|c|c|}
\hline Materiais & $\begin{array}{c}\text { Concentração de } \mathrm{H}^{+} \\
(\mathrm{mmol} / \mathrm{g})\end{array}$ & $\begin{array}{c}\text { Grupos sulfatos* } \\
(\%)\end{array}$ \\
\hline $\mathrm{SZr}$ & 9,1 & 34,0 \\
\hline $\mathrm{ZrO}_{2}$ & 1,5 & - \\
\hline CABC & 2,1 & - \\
\hline CABC/SZr-S20\% & 1,4 & 1,8 \\
\hline CABC/SZr-I20\% & 2,5 & 3,4 \\
\hline $\mathrm{CABC} / \mathrm{SZr}-\mathrm{I} 20 \% \mathrm{SC}$ & 4,5 & 10,4 \\
\hline Nióbia ${ }^{22}$ & 1,6 & - \\
\hline
\end{tabular}

* Dados obtidos por análise termogravimétrica

A acidez dos materiais foi expressa em mmol de $\mathrm{H}^{+}$por grama de catalisador. É interessante observar que o valor de acidez da SZr é cerca de 6 vezes maior do que a do óxido não sulfatado, justificando, assim, sua maior atividade catalítica. Tentando estabelecer um parâmetro para os resultados de acidez obtidos, na Tabela 2 também está presente o valor encontrado na literatura para a nióbia, ${ }^{22}$ um óxido reconhecido por suas características ácidas. Nota-se que o valor de acidez da nióbia é similar ao da zircônia, mas bastante inferior ao da zircônia sulfatada.

Um fato que chamou a atenção foi o de que o compósito obtido pelo método de síntese (CABC/SZr-S20\%) diminuiu a acidez quando comparado com o carvão. Possivelmente, no processo de síntese da SZr sobre o carvão, parte dos reagente precursores da SZr, oxicloreto de zircônio e sulfato de amônio podem ter reagido com grupos superficiais do CABC, diminuindo, assim, sua acidez.

Para o compósito CABC/SZr-I20\%, apenas um aumento de 0,4 mmol de $\mathrm{H}^{+}$também foi obtido. Por outro lado, o compósito CABC/ SZr-I20\%SC obteve uma acidez de 4,5\%. Este resultado está de acordo com o esperado, ou seja, um aumento de aproximadamente $20 \%$ foi obtido na acidez do carvão, após a impregnação da SZr. A diferença entre esses materiais é, principalmente, a etapa de calcinação realizada sob $\mathrm{N}_{2}$, a qual não está presente na síntese do $\mathrm{CABC} / \mathrm{SZr}-\mathrm{I} 20 \% \mathrm{SC}$ e interferiu na quantidade de grupos sulfatos no material.

Ainda na Tabela 2, é interessante observar que existe uma forte relação entre as variáveis acidez e teor de sulfato, o que permite inferir que a baixa acidez encontrada para CABC/SZr-S20\% e CABC/SZrI $20 \%$ está ligada à baixa quantidade de sulfatos em sua composição. 


\section{Reação de esterificação de ácido graxo - ácido láurico}

Na Figura 4a são mostrados os resultados para os diferentes catalisadores na esterificação do ácido láurico. Os resultados apresentados foram obtidos das reações desenvolvidas a $70{ }^{\circ} \mathrm{C}$, por $24 \mathrm{~h}$, 2 e $10 \%$ de $\mathrm{SZr}$ e $10 \%$ para os demais materiais (em relação à massa de ácido láurico).
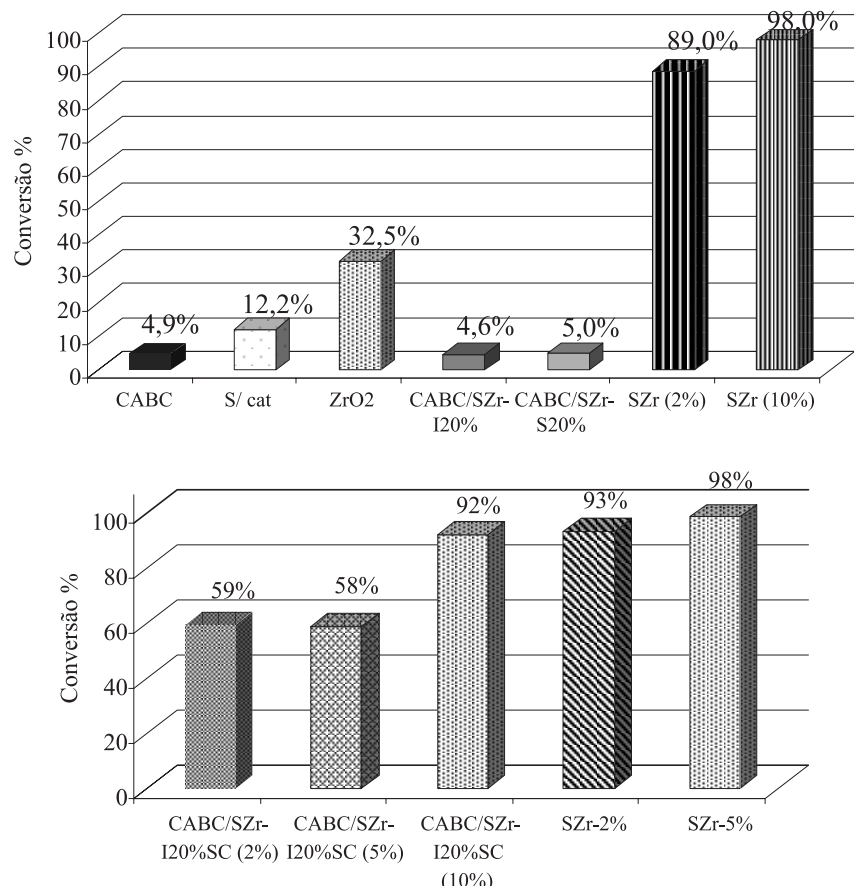

Figura 4. Rendimentos em ésteres metílicos obtidos nas reações de esterificação de ácido láurico (a) a $70{ }^{\circ} \mathrm{C}, 24 \mathrm{~h}$ sem catalisador (s/cat) e $10 \%$ dos catalisadores (SZr), (CABC), CABC/SZr-I20\%, CABC/SZr-S20\% e zircônia $\left(\mathrm{ZrO}_{2}\right)$, e (b) a $100{ }^{\circ} \mathrm{C}, 24$ h, utilizando $10 \%$ de CABC/SZr-I20\%; 2, 5 e $10 \%$ de CABC/SZr-I20\%SC e 2 e $5 \%$ de $\mathrm{SZr}$

O melhor resultado foi quando se utilizou SZr; 2 e $10 \%$ do catalisador levaram a altas conversões de ácido láurico a laureato de metila, 89 e $98 \%$, respectivamente. Para a reação desenvolvida utilizando a zircônia não sulfatada $\left(\mathrm{ZrO}_{2}\right)$, observa-se que a conversão foi de apenas $30 \%$, mostrando que a sulfatação torna a zircônia mais ativa.

Observa-se que os compósitos CABC/SZr foram pouco ativos nas reações, com resultados comparáveis ao carvão puro (CABC). Esses resultados podem ser correlacionados com a baixa acidez apresentada por esses materiais (Tabela 2).

A partir desse resultado, suspeitou-se, então, que a inatividade dos compósitos poderia estar relacionada à perda de sulfatos durante a sua síntese, na etapa de calcinação com $\mathrm{N}_{2}$ (que é utilizado na síntese dos compósitos para que não ocorra a oxidação do carvão ativado). Dessa forma, para verificar o efeito da calcinação dos compósitos nas reações, um material foi produzido pelo método de impregnação, sem a etapa de calcinação e testado nas reações (CABC/SZr-I20\% SC).

A reação realizada com este material, nas mesmas condições teve uma conversão de $34 \%$, entretanto, ainda baixa, quando comparada com os resultados obtidos utilizando $2 \%$ de $\mathrm{SZr}$ (quantidade de $\mathrm{SZr}$ presente em $10 \%$ do compósito), que foi de $89 \%$.

Alguns autores ${ }^{15,16,18}$ relataram que os melhores desempenho de catalisadores à base de SZr são encontrados em temperaturas entre $100-135^{\circ} \mathrm{C}$. Na esterificação do ácido oleico, utilizando zircônia sulfatada suportada em sílica ( $\mathrm{Zr}$ $\mathrm{SO}_{4}-\mathrm{SiO}_{2}$ ), Juan, Zhang e Yarmo ${ }^{16}$ alcançaram um máximo de conversão de $94 \%$, entretanto, utilizando temperaturas entre $110-120^{\circ} \mathrm{C}$.

Sendo assim, um teste foi realizado, variando-se as concentrações dos catalisadores $(2,5$ e $10 \%)$ e aumentando a temperatura reacional de 70 para $100{ }^{\circ} \mathrm{C}$ (Figura $4 \mathrm{~b}$ ).

A atividade catalítica do compósito CABC/SZr-I20\%SC foi intensificada com o aumento da temperatura de 70 para $100{ }^{\circ} \mathrm{C}$. As concentrações de 2 e $5 \%$ levaram a conversões similares, em torno de $60 \%$. Excelente resultado foi obtido com a utilização de $10 \%$ do I $20 \%$ SC, com conversão de $93 \%$. Ressalta-se que $10 \%$ de compósito representam $2 \%$ de SZr no meio reacional, sendo assim, baseado no resultado obtido utilizando $2 \%$ de $\mathrm{SZr}$ (93\% de conversão), pode-se inferir que o compósito apresentou atividade catalítica similar à da SZr pura.

Esses resultados mostram que a atividade catalítica dos compósitos está ligada à temperatura reacional e à acidez e, consequentemente, à quantidade de grupos sulfato presentes em sua composição.

Observa-se que o compósito calcinado (CABC/SZr-I20\%), mesmo com a temperatura reacional a $100{ }^{\circ} \mathrm{C}$, apresentou baixa conversão, confirmando que a inatividade desse material está ligada à perda de grupos sulfato durante a etapa de calcinação.

$\mathrm{O}$ aumento da temperatura reacional também resultou em uma melhora da atividade da SZr. Contudo, a atividade da SZr já é bastante elevada na temperatura de $70^{\circ} \mathrm{C}$ e o ganho em ester é pequeno. Com o aumento da temperatura, a utilização de $2 \%$ de SZr passou de 89 para $93 \%$ de conversão.

Para se verificar a dependência da atividade dos catalisadores, SZr e CABC/SZr-I20\%SC, com o tempo, as reações de esterificação foram realizadas variando-se o tempo de reação em 1, 2, 3, 4 e 6 h. As cinéticas de reação obtidas são mostradas na Figura 5.

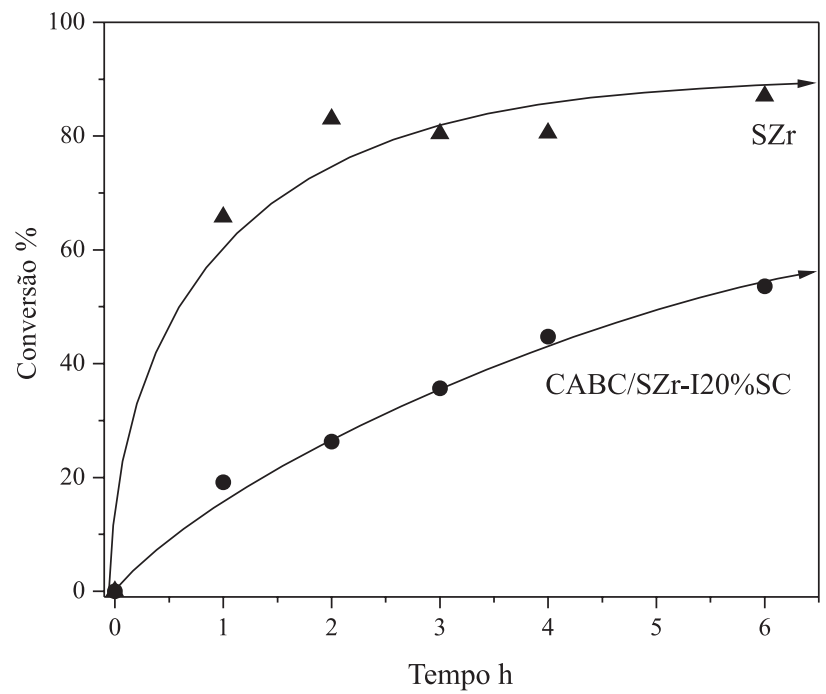

Figura 5. Cinética de esterificação do (a) ácido láurico utilizando $10 \%$ de $\mathrm{CABC} / \mathrm{SZr}-\mathrm{I} 20 \% \mathrm{SC}$ e $2 \%$ de $\mathrm{SZr}$, a $100{ }^{\circ} \mathrm{C}$

A SZr atinge em torno de $90 \%$ de conversão em, aproximadamente, 2 h (Figura 5). Para o CABC/SZr-I20\%SC, um máximo de rendimento foi alcançado com $6 \mathrm{~h}$, sendo de $53 \%$. Contudo, nota-se que, com 6 h de reação, o equilíbrio não foi atingido e uma tendência crescente pode ser observada. Devido ao fato de o carvão ativado apresentar uma estrutura microporosa e partes dos sítios ativos estarem dentro desses poros, acredita-se que, para o CABC/SZr-I20\%SC, longos tempos de reações são necessários, devido à acessibilidade e à difusão dos reagentes no catalisador.

Longos tempos de reação têm sido uma característica comum entre os catalisadores sólidos ácidos relatados na literatura. ${ }^{23,24}$ Díaz et 
$a l .{ }^{23}$ utilizaram sílica mesoporosa funcionalizada com ácido sulfônico como catalisador e obtiveram boas conversões, em torno de $80 \%$, somente depois de $8 \mathrm{~h}$ de reação. Conversões máximas, próximas a $99 \%$, foram alcançadas após $24 \mathrm{~h}$.

Apesar do compósito CABC/SZr-I20\%SC ter apresentado boas conversões na temperatura de $100{ }^{\circ} \mathrm{C}$, considerada moderada para este tipo de reação, ${ }^{3,18,25}$ e tempos de reação dentro do esperado para catalisadores sólidos ácidos, ${ }^{3,23,24}$ esse material, comparado com a SZr, não apresentou vantagens que justifiquem a sua utilização. Entretanto, um resultado importante foi obtido para o compósito: o aumento da estabilidade térmica dos grupos sulfato. Logo, busca-se uma forma mais ativa e robusta do compósito CABC/SZr-I20\%SC, partindo-se de carvões ativados com características mesoporosas e pela variação na atmosfera e temperatura de calcinação.

No processo de produção de biodiesel, ésteres monoalquílicos podem ser obtidos por meio de esterificação direta de ácidos graxos, ou por meio da esterificação e transesterificação simultânea de ácidos graxos e triglicerídeos em óleos vegetais. Atualmente, catalisadores ácidos têm sido utilizados em etapas de pré-esterificação de ácidos graxos livres presentes em óleos vegetais, para que, posteriormente, os triglicerídeos possam ser convertidos em biodiesel por meio de catálise básica, sem que ocorra a formação de grandes quantidades de sabão.

Sendo assim, o comportamento dos catalisadores foi avaliado em relação à esterificação de ácidos graxos na presença de um óleo vegetal. O óleo de soja foi utilizado como matriz e a ele adicionado $20 \%(\mathrm{~m} / \mathrm{m})$ de ácido láurico.

Observou-se que, mesmo na presença de óleo, os catalisadores seguiram a mesma tendência. A SZr melhorou seu desempenho e o equilíbrio foi atingido entre 1 e 2 h, com uma conversão de $94 \%$, tendo um pequeno incremento até $97-98 \%$, após $3 \mathrm{~h}$. Como observado anteriormente, o CABC/SZr-I20\%SC seguiu uma tendência crescente, atingindo, em 6 h, $43 \%$ de rendimento em laureato de metila.

\section{CONCLUSÃO}

Na esterificação do ácido láurico, a zircônia sulfatada apresentou um excelente desempenho, mesmo com a utilização de temperaturas mais baixas. As melhores condições para SZr foram a utilização de $2 \%$, temperatura reacional de $100{ }^{\circ} \mathrm{C}$ e $2 \mathrm{~h}$ de reação.

O compósito $\mathrm{CABC} / \mathrm{SZr}-\mathrm{I} 20 \% \mathrm{SC}$ apresentou elevada atividade, entretanto, bastante dependente do tempo reacional. Os compósitos preparados $\mathrm{CABC} / \mathrm{SZr}$-I e CABC/SZr-S não foram ativos nas reações de esterificação. A inatividade desses compósitos foi atribuída à baixa acidez, consequência da perda dos grupos sulfato durante o processo de calcinação.

\section{REFERÊNCIAS}

1. Soriano Jr., U. N.; Venditti, R.; Argyropoulos, D. S.; Fuel 2009, 88, 560.

2. Dabdoub, J. M.; Bronzel, J. L.; Rapin, M. R.; Quim. Nova 2009, 32, 776.

3. Carmo Jr., A. C.; Souza, L. K. C.; Costa, C. E. F.; Longo, E.; Zamian, J. R.; Filho, G. N. R.; Fuel 2009, 88, 461.

4. Ropero-Veja, J. L.; Aldana-Pérez, A.; Gómez, R.; Nino-Gómez, M. E; Appl. Catal., A 2010, 379, 24.

5. Grossi, C. V.; Jardim, E. O.; Araújo, M. H.;Lago, R. M.; Silva, M. J.; Fuel 2010, 89, 257.

6. López, D. E.; Goodein Jr, J. G.; Bruce, D. A.; Lotero, E.; Appl Catal., A 2005, 295, 97.

7. Ni, J.; Meunier, F. C.; Appl. Catal., A 2007, 333, 122.

8. Caetano, C. S.; Fonseca, I. M.; Ramos, A. M.; Vital, J.; Castanheiro, J. E.; Catal. Commun. 2008, 9, 1996.

9. Hino, M.; Arata, K.; Chem. Lett. 1979, 8, 477.

10. Chuah, G. K.; Jaenicke, S.; Cheong, S. A.; Chan, K. S.; Appl Catal., A 1996, 145, 267.

11. Pereira, A. L. C.; Marchetti, S. G.; Albornoz, A. Reyes, P.; Oportus, M.; Rangel, M. C.; Appl. Catal., A 2008, 334, 187.

12. Ganapati, D. Y.; Yadav, G.; Nair, J. J.; Microporous Mesoporous. Mater. 1999, 33, 1 .

13. Reddy, B. M.; Patil, M. K.; Chem. Rev. 2009, 109, 2185.

14. Vartuli, J. C.; Santiesteban, J. G.; Traverso, P.; Cardona-Martinez, N.; Chang, C. D.; Stevenson, S.; J. Catal. 1999, 187, 131.

15. Juan, J. C.; Jiang, Y.; Meng, X.; Cao. W.; Yarmo, M. A.; Zhang, J.; Mater. Res. Bull. 2007, 42, 1278.

16. Juan J. C.; Zhang, J.; Yarmo, M. A.; Appl.Catal., A 2007, 332, 209.

17. Sun, Y.; Ma, S.; Du, Y.; Yuan, L.; Wang, S.; Yang, J.; Deng, F.; Xiao, F-S.; J. Phys. Chem. B 2005, 109, 2567.

18. Garcia, C. M.; Dissertação de Mestrado, Universidade Estadual de Campinas, Brasil, 2006.

19. Du, Y.; Liu, S.; Ji, Y.; Zhang, Y.; Wei, S.; Liu, F.; Xiao, F-S.; Catal. Lett. 2008, 12, 133 .

20. Akkari, R.; Ghorbel, A.; Essayem, N.; Figueras, F.; Appl. Catal., A 2007, $328,43$.

21. Noda, L. K.; Gonçalves, N. S.; Borba, S. M.; Silveira, J. A.; Vib. Spectrosc. 2007, 44, 101

22. Carvalho, K. T. G.; Dissertação de Mestrado, Universidade Federal de Lavras, Brasil, 2009.

23. Díaz, I.; Mohino, F.; Pérez-Pariente, J.; Sastre, E.; Appl. Catal., A 2001, 205, 19.

24. Ropero-Veja, J. L.; Aldana-Pérez, A.; Gómez, R.; Nino-Gómez, M. E.; Appl. Catal., A 2010, 379, 24.

25. Mello, V. M.; Pousa, G. P. A. G.; Pereira, M. S. C.; Dias, I. M.; Suarez, P. A. Z.; Fuel Process. Technol. 2011, 92, 53. 